\title{
Stable Self-Assembled Atomic-Switch Networks for Neuromorphic Applications
}

\author{
Saurabh K. Bose, Joshua B. Mallinson, Rodrigo M. Gazoni, and Simon A. Brown
}

\begin{abstract}
Nature inspired neuromorphic architectures are being explored as an alternative to imminent limitations of conventional complementary metal-oxide semiconductor (CMOS) architectures. Utilization of such architectures for practical applications like advanced pattern recognition tasks will require synaptic connections that are both reconfigurable and stable. Here, we report realization of stable atomic-switch networks (ASN), with inherent complex connectivity, self-assembled from percolating metal nanoparticles (NPs). The device conductance reflects the configuration of synapses which can be modulated via voltage stimulus. By controlling Relative Humidity (RH) and oxygen partial-pressure during NP deposition we obtain stochastic conductance switching that is stable over several months. Detailed characterization reveals signatures of electric-field induced atomic-wire formation within the tunnel-gaps of the oxidized percolating network. Finally we show that the synaptic structure can be reconfigured by stimulating at different repetition rates, which can be utilized as short-term to long-term memory conversion. This demonstration of stable stochastic switching in ASNs provides a promising route to hardware implementation of biological neuronal models and, as an example, we highlight possible applications in Reservoir Computing (RC).
\end{abstract}

\section{Index Terms}

Atomic switch networks, Clusters, Neuromorphic architecture

\section{INTRODUCTION}

$\mathbf{T}$ HE astounding success of the von Neumann architecture for computers [1], as encapsulated in Moore's Law, is now meeting with fundamental limitations (physical transistor dimensions are approaching classical limits) and practical limitations (the exponential increase in research and development costs for every new process line) [2], [3]. Natural information processing systems, like the biological brain, on the other hand, can perform highly complex computational tasks like navigation, recognition and decision-making with remarkable ease and with very low energy consumption [4]. This natural computation, processing the useful data (patterns) from a multitude of sensory information, is immediate and cannot be matched by even the most-advanced supercomputers [5], [6]. Nature inspired architectures [7]-[13] are therefore currently being pursued as a disruptive alternative to the von Neumann architecture. A recent review on Neuromorphic architecture [14] and implementations can be found in Ref. [15].

The alternative brain-inspired hardware approach must address three key issues simultaneously: mimic the complex biological network of neurons, replicate synaptic structures and allow implementation of standard computational algorithms [16]. Achieving all of these goals is obviously enormously challenging and will require long-term research. Nevertheless significant progress has been made towards solving several different problems, using a variety of architectures. Proposals for non-CMOS approaches include those based on networks of memristors [17]-[19], atomic switches [10], [11] and Metal Oxide Resistive Random Access Memory (RRAM) [20], [21]. There have been interesting demonstrations of memristorbased neural networks [22], associative memory [23], synaptic emulators [24], conditional programming [25], reconfigurable logic [26], solving mazes [27], pattern recognition [28]-[31], and reservoir computing

The authors are with The MacDiarmid Institute for Advanced Materials and Nanotechnology, Department of Physics and Astronomy, University of Canterbury, Private Bag 4800, Christchurch 8140, New Zealand (email:simon.brown@ canterbury.ac.nz)

The authors gratefully acknowledge financial support from the Marsden Fund, New Zealand, and the MacDiarmid Institute for Advanced Materials and Nanotechnology.

This is a post-peer-review, pre-copyedit version of an article published in IEEE Trans. Elect. Dev. 2017. The final authenticated version is available online at: http://dx.doi.org/10.1109/TED.2017.2766063 

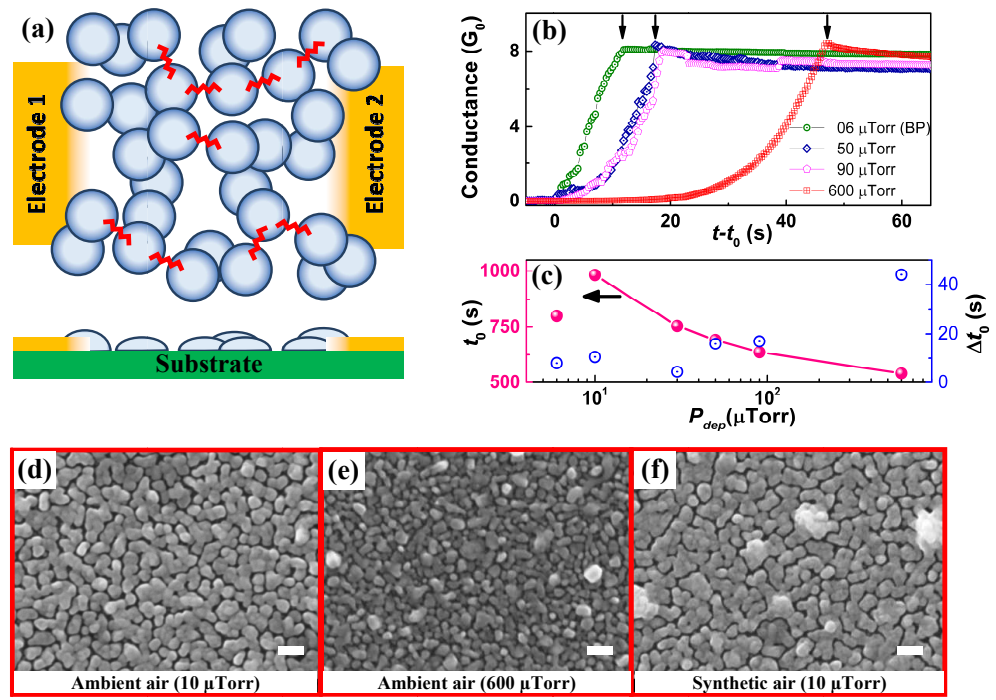

Fig. 1. (a) Schematic depiction (top and side view) of the nanoparticle network between electrical contacts of our two-electrode devices showing tunnelling gaps in the Sn NP network. The outer shaded region on the NPs depicts the thin oxide layer. (b) Variation of the onset of conductance with increase in partial pressure $P_{d e p}$ (over 2 orders of magnitude) during NP deposition. (c) This pressure variation results in shorter conduction onset times $t_{0}$ (left scale) with longer onset width $\triangle t_{0}$ (right scale). (d) Scanning electron micrograph of samples prepared at lowest ambient air pressure $\left(P_{d e p} \sim 10 \mu\right.$ Torr $)$ shows more coalescence and larger grain-size in comparison to the highest pressure (600 $\mu$ Torr) prepared samples shown in (e). (f) Use of dry synthetic air ( $P_{d e p} \sim 10 \mu$ Torr) produces a different microstructure with reduced sample stability as compared with ambient air. The white scale bars are $100 \mathrm{~nm}$.

(RC) [32]-[34]. Even in the most heavily explored architectures (regular cross-bar arrays of memristors) [35] there remain unsolved challenges in regard to realisation of the required properties of both individual switching elements and networks of these elements.

$\mathrm{RC}$ is simpler to implement than many other unconventional computation schemes since synapses do not need to be addressed individually and can be seen as an important step towards achieving other types brain-like computation. In RC a 'reservoir' comprising a complex network of switching elements allows the transformation of input signals into a higher dimensional space. [32]-[34] Training of a single 'output layer' then allows implementation of various time series prediction, pattern recognition and classification tasks. [36]-[38] Randomly assembled atomic switch networks (ASNs) based on sulphidised Ag nanowires [10], [11] and percolating films of nanoparticles [39], [40] are immediately ammenable to RC. ASNs are also an appealing alternative to regular arrays of devices because they allow realisation of complexity similar to that of the brain and fabrication via self-assembly immediately circumvent the limitations of lithographic processing. Ag-based ASNs have recently been used to demonstrate a form of $\mathrm{RC}$ in which the non-linear properties of the reservoir allows generation of target waveforms, and a clear roadmap towards further implementation has been mapped out [38].

Systems of inorganic synapses are however in the early stages of development with improvements required in production methods, reliability and actual functionality [35]. While robust switching over 10,000 cycles has been reported for $\mathrm{Ag}-\mathrm{AgS}$ nanowire systems [10] a different, and a particularly important, issue for any real-world applications is long-term device stability, which is the main focus of the present work. Such stability has not been reported previously in either Ag-AgS nanowire [10], [11], [34], [38], [41] or percolating ASNs [39].

Here we report a straightforward fabrication procedure for realization of randomly connected ASNs within a percolating network of metal nanoparticles (NPs). We show that deliberate introduction of oxygen and moisture during NP deposition leads to long term device stability. Despite the presence of oxides, the switching mechanisms associated with increases(decreases) in device conductance $G_{\uparrow}\left(G_{\downarrow}\right)$ are shown to be formation (destruction) of atomic scale wires in tunnel gaps in the network. The two-terminal device conductance $(G)$ quantifies the input-output electrode connectivity [40] and reflects the synaptic 

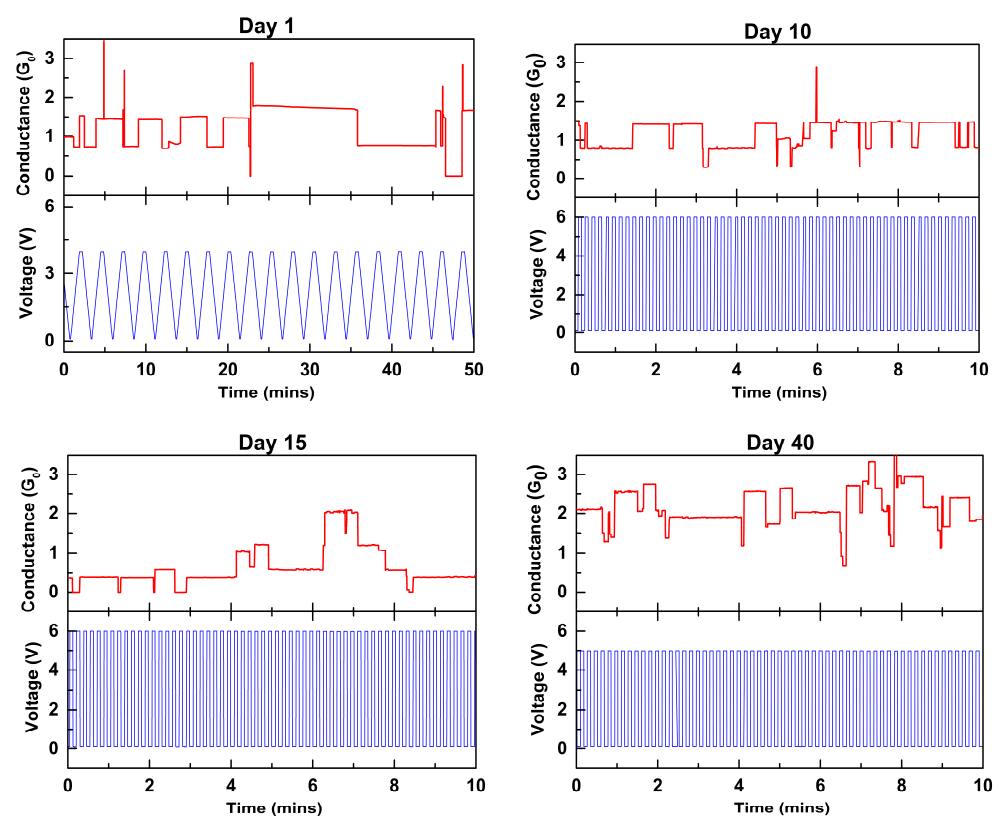

Fig. 2. Stochastic and stable switching behaviour for sample prepared with $P_{\text {dep }}=10 \mu$ Torr and high humidity (RH $\left.\sim 80 \%\right)$ ambient air conditions, showing multi-level conductance switching, induced by application of both triangular and pulsed voltage stimulus. On the $1^{\text {st }}$ day, immediately after sample fabrication, we use voltage-sweeps in order to check the voltage threshold for switching, as discussed in section IV On subsequent days we utilize controlled voltage pulses. As we tested the samples in a variety of ways at different times over a period of several months, the applied voltage in panel (d) is slightly different to that in the other panels. The sample exhibits qualitatively similar switching for several months of device operation.

configuration of the network which can be reconfigured by voltage stimulation. Finally, we discuss the observed synaptic stochasticity and why it is useful for implementation of hardware analogues of the biological brain [42].

\section{EXPERIMENTAL}

The nano-cluster deposition system used in this study is based on magnetron sputtering to generate a vapour of the metal of interest and gas aggregation to condense the vapour into particles, and has been described in detail in previous publications [43]-[45]. The deposition scheme provides a narrow cluster size distribution [43] and allows precise control over the NP surface coverage near the percolation threshold [46], so that the system is poised near criticality [47]. Sn NPs with mean diameter $8.5 \mathrm{~nm}$ are deposited between $50 \mathrm{~nm}$ thick $\mathrm{Au} / \mathrm{NiCr}$ electrodes on $\mathrm{Si}_{3} \mathrm{~N}_{4}$ substrates, with active area of $100 \mu \mathrm{m} \times$ $300 \mu \mathrm{m}$. The two-contact devices allow for a demonstration of network stability and associated dynamics, but samples with multiple contacts will be required for demonstration of RC.

The Sn NPs are deposited at room temperature which means that ordinarily the surface atoms have sufficient mobility to allow coalescence [48]. For samples poised near the percolation threshold, the coalescence can lead to the loss of conducting pathways through the film, because neighboring particles that are initially joined by a fragile connection are pulled apart as they coalesce with other neighbor NPs, thus contributing to the short life-span ( $\sim$ hours) of previous devices [39]. In the present work, the coalescence of the Sn NPs is controlled by partial oxidation (during NP deposition) via a controlled leak of air with a needle-valve. As will be shown, the controlled oxide formation leads to reduced coalescence and enhanced device stability. We emphasize that by 'stability' we do not mean that the device has a fixed conductance, but that the device is in a state in which it continues to exhibit multiple switching events in response to voltage stimuli. 


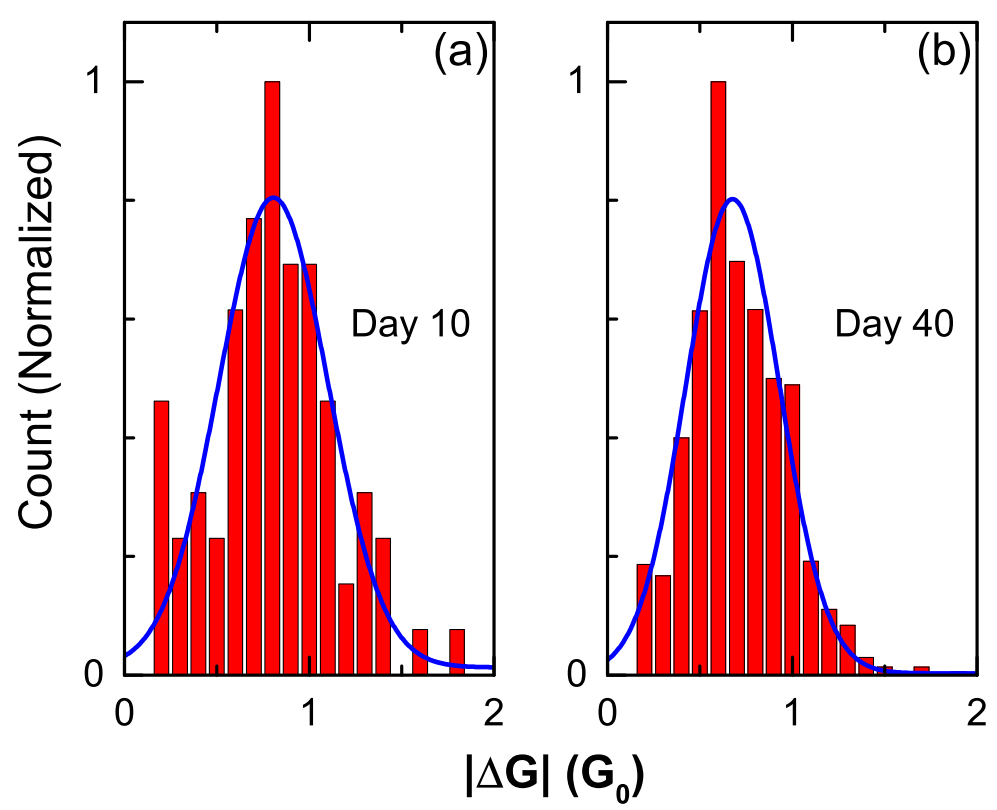

Fig. 3. The normalized distribution of the change in conductance for the switching events $(|\Delta G|)$ on day 10 and day 40 , for long periods of time, shows the stability of the switching dynamics. The solid lines are Gaussian fits to the data.

\section{SAMPLE FABRICATION}

\section{A. Self-assembly of ASNs}

We have self-assembled interconnected and active network of atomic-switches as depicted in the schematic shown in Fig. 1(a). Deposition of the Sn NPs at Base Pressure (BP, $6 \mu$ Torr) led to initial observation of a non-zero conductance (time $\left.t_{0}\right)$ at around 800s with a sharp onset behaviour $\left(\triangle t_{0}\right) \sim 10 \mathrm{~s}$, as shown in Fig. 1(b). Here, we define the width of the onset $\triangle \mathrm{t}_{0}$ as the time after $t_{0}$ required to reach a conductance of $6 G_{0}\left(G_{0}=2 e^{2} / h\right.$ is the quantum of conductance [49]). The cluster deposition is stopped [arrows in Fig. 11(b)] when $G \sim 8 G_{0}$, which represents a nanoparticle surface coverage slightly greater than the percolation threshold, and has been found experimentally to yield optimal switching behaviour when $P_{d e p}$ is in the range $\sim 10-50 \mu$ Torr, as described below. As can be seen in Fig. 11(c) the increase in deposition pressure $\left(P_{d e p}\right)$ leads to a monotonic decrease in $t_{0}$ coupled with an increase in $\triangle t_{0}$. The smooth conduction onset and longer $\triangle t_{0}$ is in contrast to the step-wise conduction onset of samples deposited at BP (minimum oxidation) in Ref. [39]; those samples were dominated by few quantized conduction pathways and therefore lacked the large-scale distributed synaptic network essential for neuromorphic applications. The post-deposition scanning electron micrographs (SEM) shown in Fig. 1(d-e) reveal markedly smaller NP sizes for devices prepared at higher $P_{d e p}$. This can be understood in the framework of diffusion of the tin NPs on the substrate being inhibited by the formation of tin-oxide-shell, leading to reduced coalescence at higher $P_{d e p}$. Such formation of oxide-shell is known to inhibit grain-rotation-induced grain coalescence (GRIGC) [50] thus favoring smaller grain-sizes. This reduced coalescence means that the percolation threshold is reached more quickly ( $\operatorname{smaller} t_{0}$ ) and the conductance then increases more slowly (larger $\left.\triangle t_{0}\right)$. After the deposition is stopped [arrows in Fig. 11(b)] the slow conductance change is primarily due a small amount of further coalescence of the NPs, decreasing $G$.

\section{B. Optimization of Pressure and Humidity}

The samples fabricated with high Relative Humidity $(\mathrm{RH} \sim 80 \%)$ ambient air have been stimulated with voltage sweeps and square voltage pulses over several months. Data for a typical sample are shown in Fig. 2. The four panels show representative snapshots of the switching events on the $1^{\text {st }}, 10^{\text {th }}, 15^{\text {th }}$ and $40^{t h}$ day. As described below, the detailed switching behaviour of the network is a complex function 


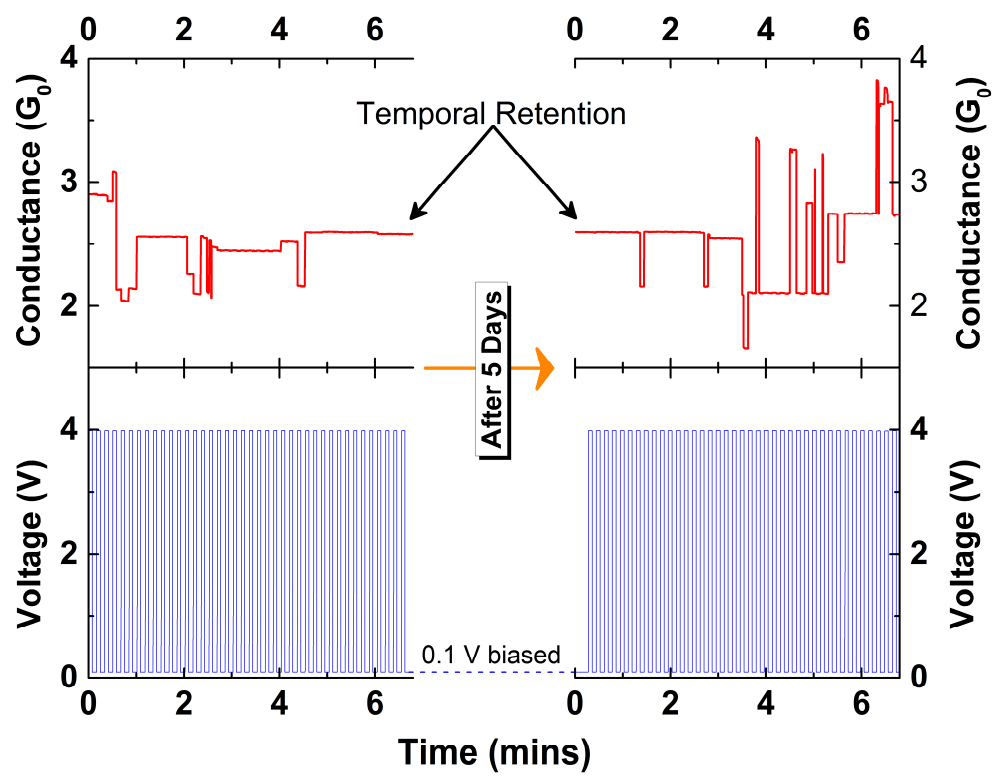

Fig. 4. Temporal retention of the network conductance tested over more than one week. Left: the device shows switching in response to $4 \mathrm{~V}$ pulses before a constant 'read' voltage $(0.1 \mathrm{~V})$ was applied for 5 days. Right: the conductance showed no measurable change and switching re-commenced on application of $4 \mathrm{~V}$ pulses again.

of applied voltage, pulse widths $\left(\tau_{p}\right)$ and history of the inputs, but qualitatively similar conductance switching is observed for long periods of time. Application of voltage sweep or pulses induces Electric Field Induced Evaporation (EFIE) and Electric Field Induced Surface Diffusion (EFISD) of the surface atoms [51], resulting in atomic-wire formation in tunnel gaps in the percolating network (resulting in $G_{\uparrow}$ ). The electronic flow causes electromigration induced opening of the previously connected atomic-wires [52] resulting in $G_{\downarrow}$. The conductance thus switches between multiple conductance states with $G \sim 1-$ $3 G_{0} . G \rightarrow 0$ at multiple points, but the electric field induces reconnections and results in the network configuration returning to the connected regime of non-zero conductance. Samples fabricated in high humidity conditions ( $\mathrm{RH} \sim 80 \%$ ) with ambient air have been tested in this way for periods of several months without the sample becoming permanently open circuit. The key point is that the samples prepared with high RH oxidation exhibit stable switching behaviour without significant performance degradation. For example, Fig. 3 shows the distribution of the change in conductance for switching events $(|\Delta G|)$ on day 10 and 40 (see Fig. 2). Both the mean and variance of the distributions are very similar, indicating that the average switching behavior is the same. Obviously it is an onerous task to test such samples for much longer periods and further testing is required to determine the ultimate lifetime of the samples.Both the mean and variance of the distributions are very similar. Obviously it is an onerous task to test such samples for much longer periods and further testing is required to determine the ultimate lifetime of the samples. The same kind of stable switching behaviour is observed for voltages upto $(\geq 7-8 \mathrm{~V})$ but application of very high voltages $(\geq 10 \mathrm{~V})$ causes irreversible breakdown in the devices.

The oxidation of pure $\mathrm{Sn}$ into tin-oxides $\left[\mathrm{SnO}, \mathrm{SnO}_{2}\right]$ is well known to be accelerated under humid conditions. [53] Studies of oxidation of $\mathrm{Sn}$ in conditions similar to the present ones show that only partial surface oxides are formed. [54], [55] Ex-situ analysis of the present oxide-structure is obviously not feasible and so instead we have investigated in-situ the device stability over several weeks. Fig. 4 shows that the device conductance did not change measurably when the device was left for 5 days with $0.1 \mathrm{~V}$ applied. The conductance switching is resumed when voltage pulses were applied again, which clearly indicates that the oxide structure did not evolve significantly in this period.

The final microstructure and the associated device stability achieved with NP oxidation depends strongly on the relative humidity during NP deposition. Therefore, in order to develop a reliable fabrication process, mitigating day-to-day variation in $\mathrm{RH}$ of the ambient air and to build understanding of the critical $\mathrm{RH}$ 

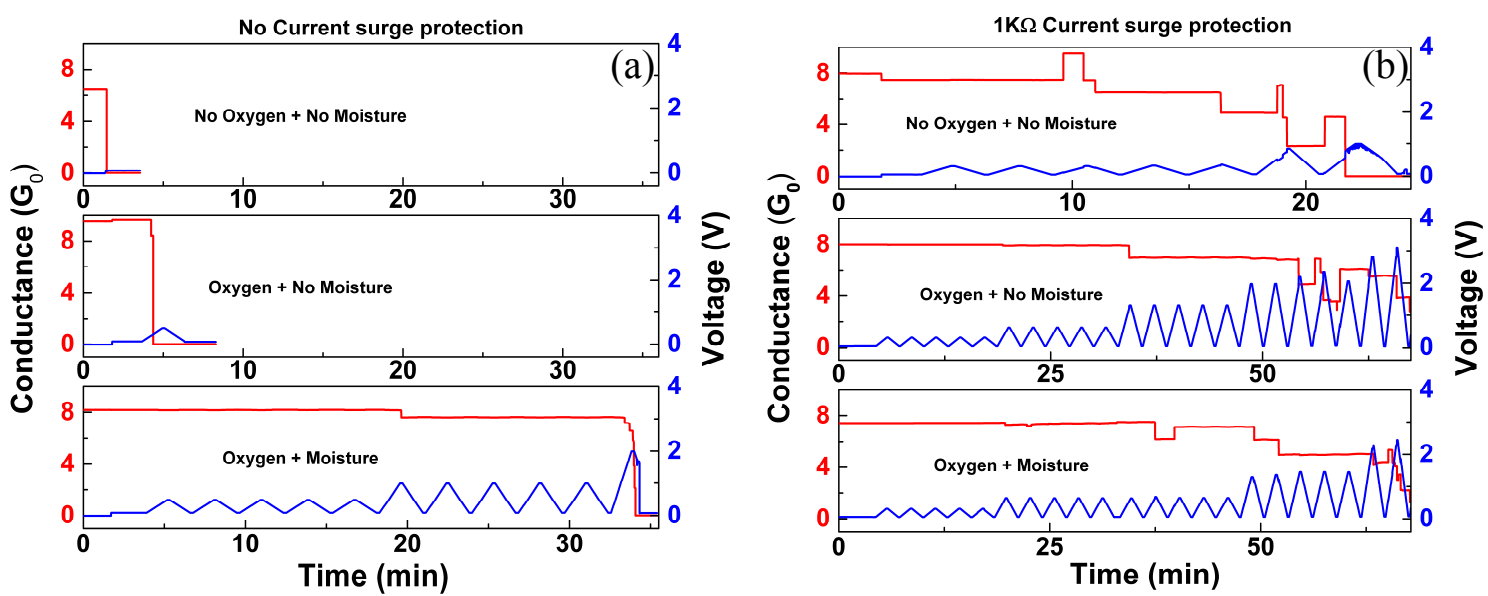

Fig. 5. Samples prepared with $P_{d e p}=10 \mu$ Torr and sub-optimum moisture content $(\mathrm{RH} \sim 55 \%)$ can be partially stabilized with a currentsurge protection in the form of a $1 \mathrm{k} \Omega$ in-line resistor. The top panels in (a) and (b) show the samples prepared without oxidation or moisture are unstable even with the current surge protection. The samples become open circuit $(G \rightarrow 0)$ on application of $0.1 \mathrm{~V}$ (no resistor) and $1 \mathrm{~V}$ (with resistor). The middle panel in (a) shows that samples prepared with dry oxygen (no moisture) are only slightly more stable as $G \rightarrow 0$ at $1 \mathrm{~V}$ (no resistor). The equivalent sample with in-line resistor showed stability in the data shown here but $G \rightarrow 0$ in the next measurement (not shown here). The bottom panels show that samples prepared in oxygen and partial humidity $(\sim 55 \%)$ are more stable than those in the middle panels (no moisture). The sample prepared with oxygen + moisture and measured with $1 \mathrm{k} \Omega$ resistor showing $G$ switching for several weeks.

necessary for stabilization, a new set of samples were prepared in a more controlled environment using commercial dry synthetic air coupled with custom-built humidifier (bubbler). The deposition with dry synthetic air resulted in unstable samples with the corresponding SEM micrographs [shown in 11(f)] indicating a slightly more coalesced morphology in comparison to the RH $\sim 80 \%$ ambient air in 1 (d). Although the microstructure has only subtle differences, these differences become very important as the NP system is poised near the percolation threshold and nanoscale changes can promote(inhibit) inter-NP atomic-switch formation.

\section{Current surge protection}

The variation in conductance during the first voltage sweeps applied to the new series of samples are shown in Fig. 5. The top panel of Fig. 5(a) shows that the atomic switch networks prepared at BP with no oxygen and no moisture are disconnected on application of very small voltages of $0.1 \mathrm{~V}$. Introduction of dry air at $P_{d e p}=10 \mu$ Torr, no moisture [Fig. 5(a) middle panel] leads to samples that can only sustain small voltage sweeps. Samples prepared with the same $P_{d e p}=10 \mu$ Torr and higher RH $\sim 55 \%$ are more stable but still do not show sustained reconnections $\left(G_{\uparrow}\right)$ and become disconnected at $\sim 2 \mathrm{~V}$, indicating that these samples are only partially stabilized. This provides an opportunity to demonstrate an additional method for stabilizing the switching behaviour in these devices.

Fig. 5(b) shows that the devices show more stable conductance switching when measured with a currentlimiting resistor $(1 \mathrm{k} \Omega)$ in series with the device. The series resistor limits the maximum allowed current flowing through the percolating network and hence prevents the destruction of the key connections via electromigration. The sample prepared with $P_{d e p}=10 \mu$ Torr but without moisture and measured with the series resistor survived the voltage sweeps [Fig. 5(b) middle panel], but got disconnected in the next measurement (not shown here). In contrast, the sample prepared with oxidation with $\mathrm{RH} \sim 55 \%$ and measured with the in-line resistor was stable for several weeks. Further samples prepared in synthetic air with a higher RH ( $\sim 60 \%)$ exhibit stable (i.e. for months) switching behaviour as in Fig. 2, without a current-limiting resistor. This indicates that oxidation with a critical amount of moisture $\mathrm{RH}(\geq 60 \%)$ creates a microstructure which incorporates a robust current-limiting resistor backbone, and thus do not require additional in-series resistor protection. 


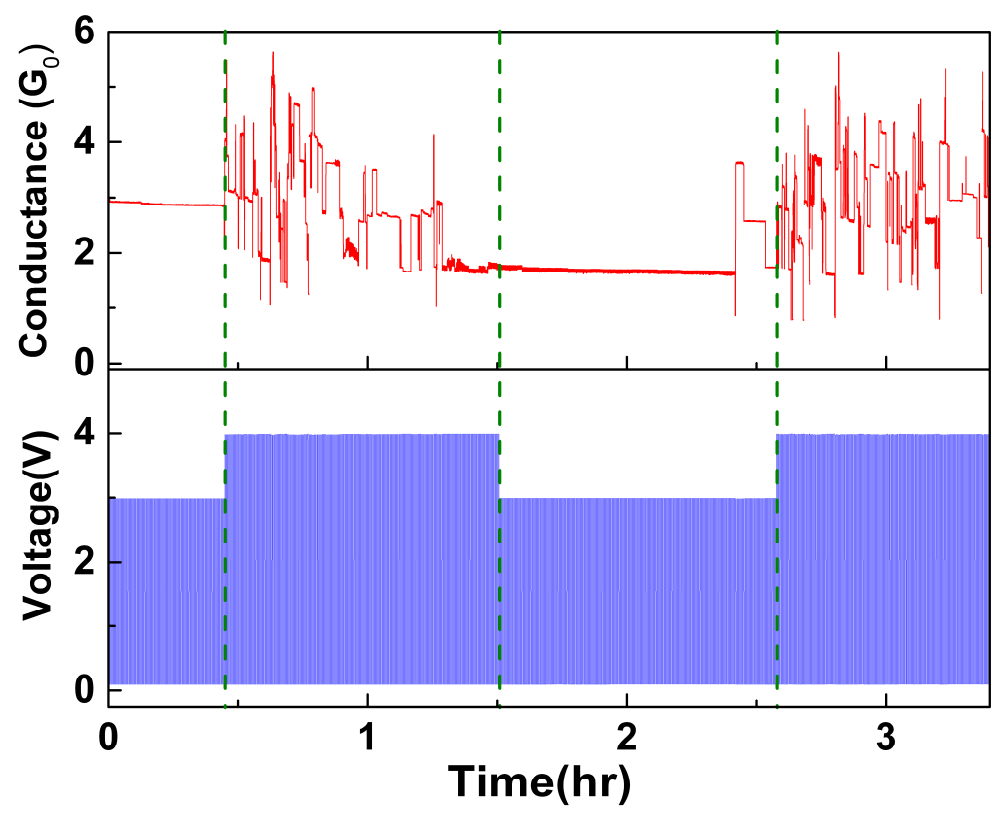

Fig. 6. Further conductance data of the sample described in Fig. 2 showing that switching in these NP assemblies requires application of a minimum voltage-stimulus. The $3 \mathrm{~V}$ pulses generate very few switching events, whereas $4 \mathrm{~V}$ pulses trigger multitude of switching events.

\section{Fabrication summary}

As discussed above, the crucial fabrication parameters for ASN stability are $P_{d e p}$ and relative humidity RH\%. $P_{d e p}$ was varied between BP (6 $\mu$ Torr) and $600 \mu$ Torr, and RH\% was varied from completely dry $(0 \%)$ to nearly saturated $(80 \%)$. The optimal fabrication parameters for stability of these Sn cluster devices are $P_{d e p}=10-50 \mu$ Torr and relative humidity $\mathrm{RH}=60-80 \%$ when an in-series resistor of $1 \mathrm{k} \Omega$ is used for current surge protection.

\section{SWITCHING MECHANISM AND DYNAMICS}

To understand the physical process underlying the switching mechanism, we present further voltage and time dependent studies. Fig. 6 presents a segment of data acquired during the long sequence of measurements on the sample used to obtain the data in Fig. 2 (ambient air, $P_{d e p}=10 \mu$ Torr, RH $\sim$ $80 \%$ ) showing that a critical voltage (or equivalently, electric field) is required to activate the switching process. The switching dynamics is voltage polarity independent, with negative $\mathrm{V}$ pulses (not shown here) showing exactly the same switching dynamics as positive $\mathrm{V}$ pulses. This polarity-independence allows us to eliminate other possible switching mechanisms such as Coulomb charging and electrochemical redox reactions [10], and further substantiates the electric-field and current induced switching mechanism described here. As shown in Fig. 6, the pulses with amplitude 3V cause almost no switching events, whereas $4 \mathrm{~V}$ pulses induce multiple stochastic switching events. The inherently probabilistic nature of the synaptic connections are clearly visible in the snapshots shown in Fig. 2, where stimulus near the threshold voltage induces less than one switching event per pulse. Such stochastic or probabilistic dynamics of the synapses are integral to the functioning of the biological brain: e.g. the opening and closing of synaptic ion channels and associated transmission of neurotransmitter molecules is inherently stochastic [42] and is understood to be critical for noise-filtering [56], signal transmission [7] and rewardmodulated Hebbian learning [57]. The existence of a critical stimulus strength (electric field here) for the stochastic formation (annihilation) of atomic-scale wires in tunnel gaps in the network is consistent with EFIE/EFISD (electromigration) mechanisms and provides a unique global control over the synaptic network reconfiguration.

To further validate the model and estimate the effective tunnel barrier parameters associated with the gap in which the atomic wires are formed, pulsed voltage measurements were stopped when the device 

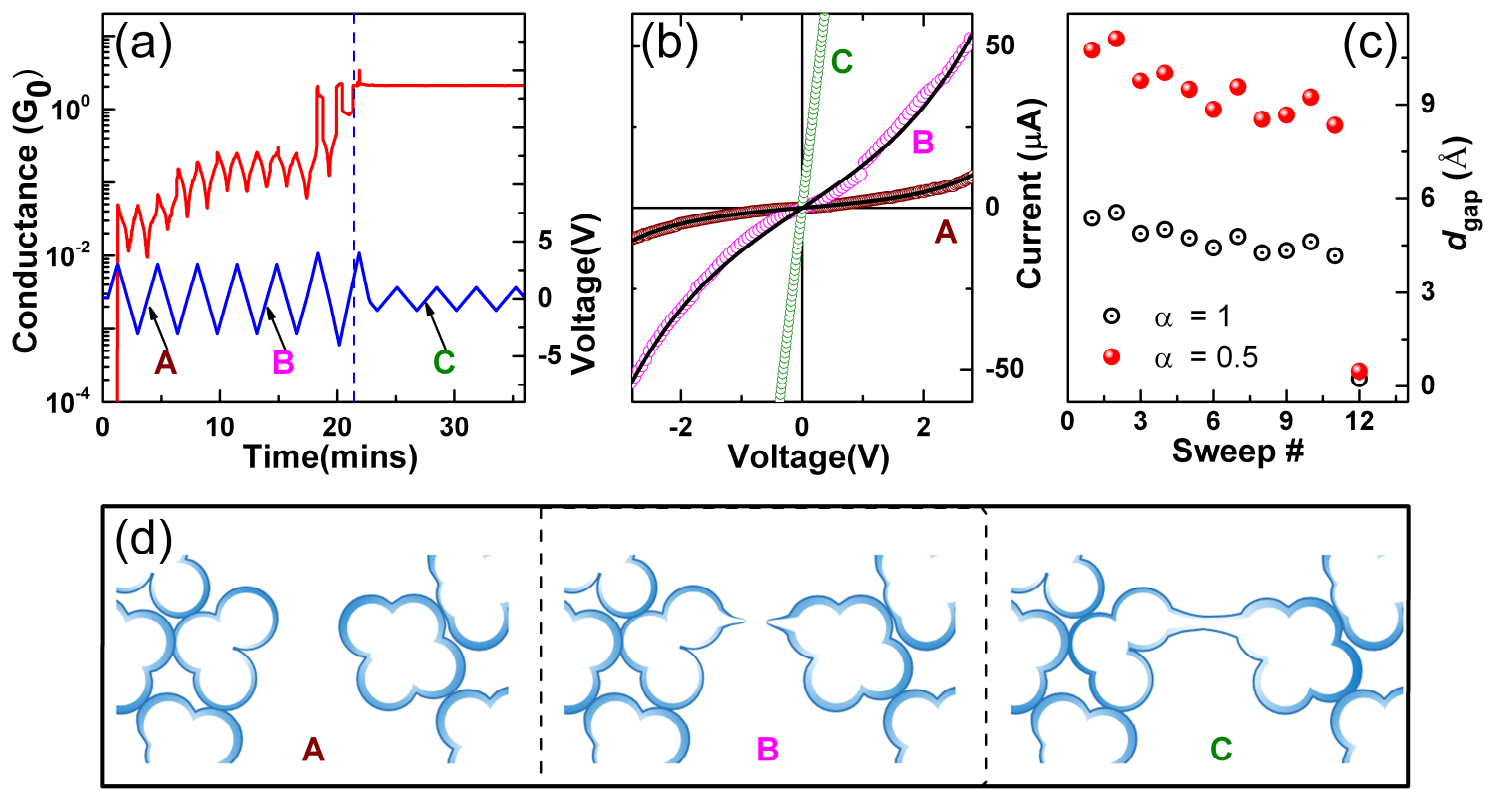

Fig. 7. (a-b) Slow bipolar voltage sweeps were applied to the sample described in Fig. 2 when $G=0$. The tunneling behaviour is evident from the non-linear current-voltage $I(V)$ characteristics e.g. at points A and B. Successive voltage-sweeps increase $G$ by two orders of magnitude before the jump-to-contact and $G \rightarrow 2 \mathrm{G}_{0}$ at $\sim 22 \mathrm{~min}$. This newly formed connection is then stable under further voltage sweeps and shows the expected linear $I(V)$ characteristics. The solid lines in panel (b) are fits to Simmons' tunneling model (1). (c) The calculated tunnel gap $d_{\text {gap }}$ decreases monotonically before an ohmic connection is formed at sweep 12 . The $I(V)$ is ohmic after that (e.g. position C). (d) Schematic depicting the gradual formation of atomic wire in one of the tunnel barrier.

became open circuit (i.e. $G<10^{-5} G_{0}$ ). A series of slow bipolar voltage sweeps were then applied which showed non-linear current-voltage $I(V)$ characteristics as in Fig. 7 (a-b). On the $1^{\text {st }}$ voltage sweep, $G$ jumps from $<10^{-5} G_{0}$ to $\sim 10^{-2} G_{0}$, which corresponds to the formation of a tunnel gap which is sufficiently small to allow a measurable current to flow. The corresponding tunneling current through a non-ideal potential barrier with height $\Phi_{B}$ and width $d$ is [58]:

$$
\begin{aligned}
I \propto\left(\Phi_{B}-e V / 2\right) \exp \left[-\frac{2(2 m)^{1 / 2}}{\hbar} \alpha\left(\Phi_{B}-e V / 2\right)^{1 / 2} d\right] \\
-\left(\Phi_{B}+e V / 2\right) \exp \left[-\frac{2(2 m)^{1 / 2}}{\hbar} \alpha\left(\Phi_{B}+e V / 2\right)^{1 / 2} d\right]
\end{aligned}
$$

with $m$ being the free electron mass and $\alpha$ being an adjustable parameter representing the nonideal character of the tunneling barrier and effective electron mass. Fig. 7(b) shows representative $I(V)$ characteristics for the voltage sweeps marked A, B and C in Fig. 7) (a) along with the fits to (1) (solid lines). The associated barrier width $d$, with calculated barrier height $\Phi_{B} \sim 2 \mathrm{eV}$, decreases monotonically with the sweep number \#, when either an ideal $(\alpha=1)$ or highly non-ideal $(\alpha=0.5)$ barrier is assumed. Interestingly the resultant electric field exceeds the $\sim 1 \mathrm{Vnm}^{-1}$ threshold for EFISD but remains lower than the $\sim 25 \mathrm{Vnm}^{-1}$ required for EFIE [39], [51]. As shown schematically in Fig. 7(d), the narrowing of the tunnel gap under the influence of the electric field continues, until after about 20 minutes an atomic scale wire closes the tunnel gap, i.e. a "jump to contact" occurs [59] leading to a conductance $G=2 G_{0}$. These atomic-scale wires are similar to those formed in mechanically controlled break junctions (MCBJs) [59]. The wire breaks and re-forms a couple of times [Fig. 7(a)] and then is observed to be completely stable when subjected to further voltage sweeps. The Ohmic conductance is marked by linear $I(V)$ behavior depicted in curve $\mathrm{C}$ of Fig. 7(b). Such conductance modulation with successive stimulus (electric-field) is one of the key requirements for synaptic learning capability in neuromorphic systems [40], [60], and is similar to the sensory memory reported in Ref. [61]. 

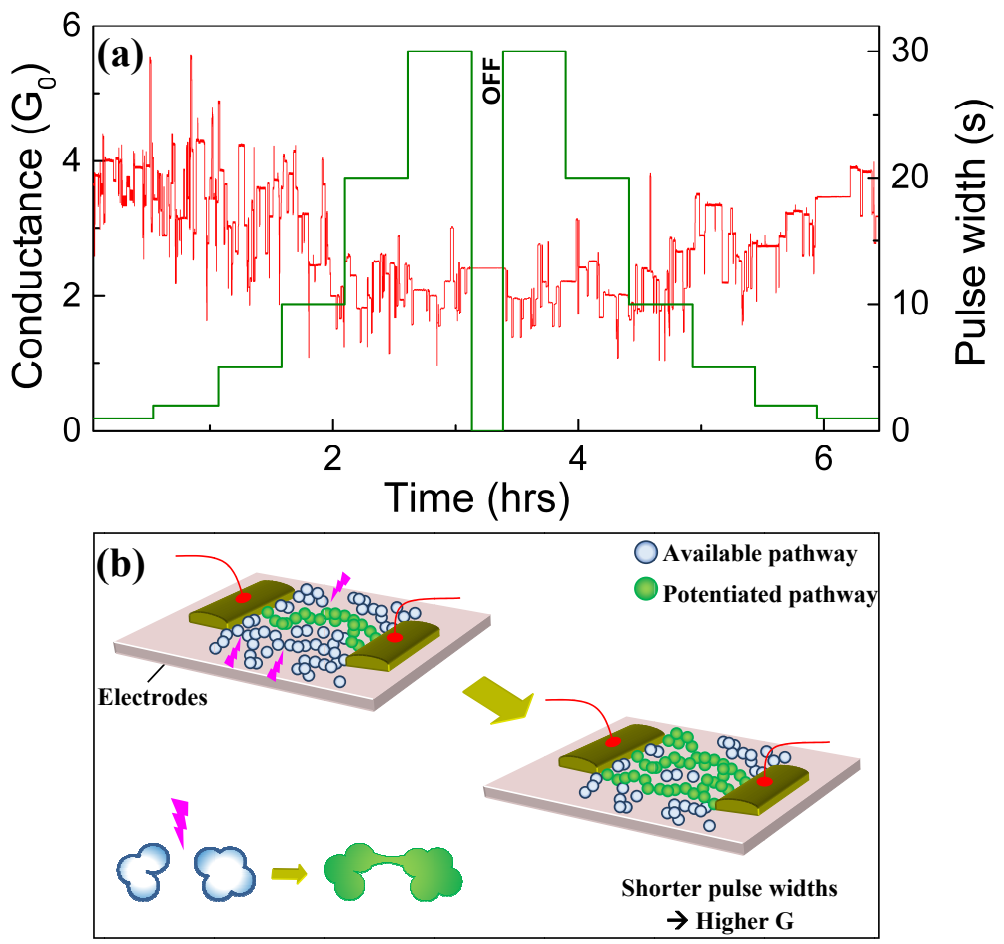

Fig. 8. (a) Synaptic plasticity dependence on stimulus frequency is depicted in switching behaviour in response to a sequence of voltage pulses with fixed $V_{p}=4 \mathrm{~V}$ and variable pulse widths $\tau_{p}(1-30 \mathrm{~s})$. The conductance remains unaltered for read voltages of $0.1 \mathrm{~V}$ as seen in the flat section (OFF) in middle of the sequence. (b) Schematic depiction of representative synaptic pathways with only very few pathways shown for clarity. The real device ASN is much more complex. Shorter pulse widths leads to electric-field induced connections as described in Fig. 7 Formation of additional atomic-wire connections (one depicted here) cause more potentiated synaptic pathways and thus higher conductance as seen in panel (a).

Realization of neuromorphic behavior in these ASNs also requires a scheme to modify the density of potentiated synaptic pathways [40]. In Fig. 8 we show such stimulus frequency dependent potentiation. Square voltage pulses with fixed $V_{p}=4 \mathrm{~V}$ (just above threshold voltage) with various pulse widths $\tau_{p}(1$, 2, 5, 10, 20, and $30 \mathrm{~s}$ ) are applied successively for $30 \mathrm{mins}$ each (the green lines depict the pulsewidth). Longer $\tau_{p}$ (slow pulses) leads to lower conductance whereas shorter $\tau_{p}$ (faster pulses) leads to additional formation of synaptic pathways as schematically depicted in Fig. 8(b), resulting in higher $G$. This variation can be understood in the light of the electric field-induced reconnections dominating over the electromigration induced disconnection of atomic wires for shorter $\tau_{p}$. Such stimulus rate dependent reconfiguration of network connectivity can be modeled as short-term to long-term memory conversion [12], [62].

\section{CONCLUSION}

In summary, we have demonstrated a unique approach for realization of self-assembled atomic switch networks with stimulus induced control of the synaptic configuration reflected in the device conductance. By controlling oxidation and humidity during NP deposition, nanoparticle coalescence is inhibited resulting in stochastic switching that is stable over several months. The atomic-wire formation in these oxidised nanostructures is very surprising and detailed modeling [63] of the atomistic mechanisms [39], [51], [52] in the presence of oxides [54], [55] is required, as is atomic scale modelling of the effect of humidity [53] on the oxidation process.

We have also highlighted the stochastic nature of the switching mechanism together with the stability of the distribution of switching events - these reflect inherently complex network dynamics that are a key requirement for neuromorphic applications. The next stage of this research will be to build devices with multiple contacts and to demonstrate that the networks exhibit the required network dynamics. The 
precise requirements are different for different applications [15], [35], but for RC include distributed spatio-temporal dynamics, recurrency, higher harmonic generation, switching speed, network size and type of connectivity [10], [32], [33], [38], [64], [65].

More generally, these complex percolating structures mimic some of the features of biological neural networks and synaptic structures and so could provide a foundation for a range of future neuromorphic architectures. Future work will focus on utilizing these structures to implement previously suggested algorithms [32], [33] and hence to demonstrate utility in key applications.

\section{ACKNOWLEDGMENT}

\section{The technical support of G. MacDonald and G. Graham are acknowledged.}

\section{REFERENCES}

[1] H. H. Goldstine and Neumann Von. J., "On the principles of large scale computing machines," John von Neumann Collect. Work., vol. 5, pp. 1-32, 1946.

[2] Y. Taur, D. A. Buchanan, W. Chen, D. J. Frank, K. E. Ismail, L. Shih-Hsien, G. A. Sai-Halasz, R. G. Viswanathan, H. J. C. Wann, S. J. Wind, and H. S. Wong, "CMOS scaling into the nanometer regime," Proc. IEEE, vol. 85, no. 4, pp. 486-503, 1997.

[3] D. J. Frank, "Power-constrained CMOS scaling limits," IBM J. Res. Dev., vol. 46, no. 2.3, pp. 235-244, 2002.

[4] E. R. Kandel, J. H. Schwartz, and J. M. Jessell, Principles of Neural Science 4th edn. McGraw-Hill, 2000.

[5] T. Roska, "Cellular Wave Computers for Brain-Like Spatial-Temporal Sensory Computing," IEEE Circuits Syst. Mag., vol. 5, pp. 5-19, 2005.

[6] T. M. Wong, R. Preissl, P. Datta, M. D. Flickner, R. Singh, S. K. Esser, E. McQuinn, R. Appuswamy, W. P. Risk, H. D. Simon, and D. S. Modha, "IBM Research Report 10^14," IBM Res. Rep. 10^14, vol. 10502, pp. 1-3, 2012.

[7] T. Tuma, A. Pantazi, M. Le Gallo, A. Sebastian, and E. Eleftheriou, "Stochastic phase-change neurons," Nat. Nanotechnol., vol. 11, no. 8, pp. 693-699, 2016.

[8] S. K. Bose, C. P. Lawrence, Z. Liu, K. S. Makarenko, R. M. J. van Damme, H. J. Broersma, and W. G. van der Wiel, "Evolution of a designless nanoparticle network into reconfigurable Boolean logic," Nat. Nanotechnol., vol. 10, no. 12, pp. 1048-1052, 2015.

[9] R. F. Service, "The brain chip," Science, vol. 345, no. 6197, pp. 614-616, 2014.

[10] A. V. Avizienis, H. O. Sillin, C. Martin-Olmos, H. H. Shieh, M. Aono, A. Z. Stieg, and J. K. Gimzewski, "Neuromorphic atomic switch networks." PLoS One, vol. 7, no. 8, p. e42772, 2012.

[11] A. Z. Stieg, A. V. Avizienis, H. O. Sillin, C. Martin-Olmos, M. Aono, and J. K. Gimzewski, "Emergent criticality in complex turing B-type atomic switch networks," Adv. Mater., vol. 24, pp. 286-293, 2012.

[12] T. Ohno, T. Hasegawa, T. Tsuruoka, K. Terabe, J. K. Gimzewski, and M. Aono, "Short-term plasticity and long-term potentiation mimicked in single inorganic synapses." Nat. Mater, vol. 10, no. 8, pp. 591-595, 2011.

[13] M. Suri, D. Querlioz, O. Bichler, G. Palma, E. Vianello, D. Vuillaume, C. Gamrat, and B. Desalvo, "Bio-inspired stochastic computing using binary CBRAM synapses," IEEE Trans. Electron Devices, vol. 60, no. 7, pp. 2402-2409, 2013.

[14] C. Mead, "Neuromorphic Electronic Systems," Proc. IEEE, vol. 78, no. 10, pp. 1629-1636, 1990.

[15] R. A. Nawrocki, R. M. Voyles, and S. E. Shaheen, "A Mini Review of Neuromorphic Architectures and Implementations," IEEE Trans. Electron Devices, vol. 63, no. 10, pp. 3819-3829, 2016.

[16] D. Sterratt, B. P. Graham, A. Gillies, and D. J. Willshaw, Principles of Computational Modelling in Neuroscience. Cambridge Univ. Press, 2011.

[17] G. Indiveri, B. Linares-Barranco, R. Legenstein, G. Deligeorgis, and T. Prodromakis, "Integration of nanoscale memristor synapses in neuromorphic computing architectures." Nanotechnology, vol. 24, no. 38, p. 384010, 2013.

[18] D. Querlioz, O. Bichler, P. Dollfus, and C. Gamrat, "Immunity to device variations in a spiking neural network with memristive nanodevices," IEEE Trans. Nanotechnol., vol. 12, no. 3, pp. 288-295, 2013.

[19] G. C. Adam, B. D. Hoskins, M. Prezioso, F. Merrikh-Bayat, B. Chakrabarti, and D. B. Strukov, "3-D Memristor Crossbars for Analog and Neuromorphic Computing Applications," IEEE Trans. Electron Devices, vol. 64, no. 1, p. 312, 2017.

[20] S. Yu, Y. Wu, R. Jeyasingh, D. Kuzum, and H. S. P. Wong, "An electronic synapse device based on metal oxide resistive switching memory for neuromorphic computation,” IEEE Trans. Electron Devices, vol. 58, no. 8, pp. 2729-2737, 2011.

[21] J. Park, M. Kwak, K. Moon, J. Woo, D. Lee, and H. Hwang, "TiOx-Based RRAM Synapse With 64-Levels of Conductance and Symmetric Conductance Change by Adopting a Hybrid Pulse Scheme for Neuromorphic Computing," IEEE Electron Device Lett., vol. 37 , no. 12 , pp. $1559-1562,2016$.

[22] A. Thomas, "Memristor-based neural networks," J. Phys. D Appl. Phys., vol. 46, p. 093001, 2013.

[23] Y. V. Pershin and M. Di Ventra, "Experimental demonstration of associative memory with memristive neural networks," Neural Networks, vol. 23, no. 7, pp. 881-886, 2010.

[24] Z. Wang, S. Joshi, S. E. Savel'ev, H. Jiang, R. Midya, P. Lin, M. Hu, N. Ge, J. P. Strachan, Z. Li, Q. Wu, M. Barnell, G.-L. Li, H. L. Xin, R. S. Williams, Q. Xia, and J. J. Yang, "Memristors with diffusive dynamics as synaptic emulators for neuromorphic computing," Nat. Mater, vol. 16, pp. 101-108, 2017.

[25] J. Borghetti, Z. Li, J. Straznicky, X. Li, D. A. A. Ohlberg, W. Wu, D. R. Stewart, and R. S. Williams, "A hybrid nanomemristor/transistor logic circuit capable of self-programming." Proc. Natl. Acad. Sci. U. S. A., vol. 106, no. 6, pp. 1699-1703, 2009. 
[26] Q. Xia, W. Robinett, M. W. Cumbie, N. Banerjee, T. J. Cardinali, J. J. Yang, W. Wu, X. Li, W. M. Tong, D. B. Strukov, G. S. Snider, G. Medeiros-Ribeiro, and R. S. Williams, "Memristor-CMOS hybrid integrated circuits for reconfigurable logic," Nano Lett., vol. 9, no. 10, pp. 3640-3645, 2009.

[27] Y. V. Pershin and M. Di Ventra, "Solving mazes with memristors: A massively parallel approach," Phys. Rev. E - Stat. Nonlinear, Soft Matter Phys., vol. 84, no. 4, pp. 1-6, 2011.

[28] G. S. Snider, "Self-organized computation with unreliable, memristive nanodevices," Nanotechnology, vol. 18, p. $365202,2007$.

[29] F. Alibart, E. Zamanidoost, and D. B. Strukov, "Pattern classification by memristive crossbar circuits using ex situ and in situ training." Nat. Commun., vol. 4, no. May, p. 2072, 2013.

[30] M. Chu, B. Kim, S. Park, H. Hwang, M. Jeon, B. H. Lee, and B. G. Lee, "Neuromorphic Hardware System for Visual Pattern Recognition with Memristor Array and CMOS Neuron," IEEE Trans. Ind. Electron., vol. 62, no. 4, pp. 2410-2419, 2015.

[31] P. Sheridan, W. Ma, and W. Lu, "Pattern recognition with memristor networks," Proc. - IEEE Int. Symp. Circuits Syst., pp. 1078-1081, 2014.

[32] Z. Konkoli and G. Wendin, “On Information Processing with Networks of Nano-Scale Switching Elements," Int. Journ. Unconv. Comput., vol. 10, pp. 405-428, 2014.

[33] M. S. Kulkarni and C. Teuscher, "Memristor-based Reservoir Computing," in IEEE/ACM Inter- Natl. Symp. Nanoscale Archit., 2012, p. 226.

[34] H. O. Sillin, R. Aguilera, H.-H. Shieh, A. V. Avizienis, M. Aono, A. Z. Stieg, and J. K. Gimzewski, "A theoretical and experimental study of neuromorphic atomic switch networks for reservoir computing." Nanotechnology, vol. 24, p. $384004,2013$.

[35] G. W. Burr, R. M. Shelby, A. Sebastian, S. Kim, S. Kim, S. Sidler, K. Virwani, M. Ishii, P. Narayanan, A. Fumarola, L. L. Sanches, I. Boybat, M. Le Gallo, K. Moon, J. Woo, H. Hwang, and Y. Leblebici, "Neuromorphic computing using non-volatile memory," Adv. Phys. X, vol. 2, no. 1, pp. 89-124, 2017.

[36] C. Fernando and S. Sojakka, "Pattern Recognition in a Bucket." Springer, Berlin, Heidelberg, 2003, pp. 588-597.

[37] S. Choi, P. Sheridan, and W. D. Lu, "Data Clustering using Memristor Networks," Sci. Rep., vol. 5, no. 1, p. $10492,2015$.

[38] E. C. Demis, R. Aguilera, K. Scharnhorst, M. Aono, A. Z. Stieg, and J. K. Gimzewski, "Nanoarchitectonic atomic switch networks for unconventional computing," Jpn. J. Appl. Phys., vol. 55, no. 11, p. 1102B2, 2016.

[39] A. Sattar, S. Fostner, and S. A. Brown, "Quantized conductance and switching in percolating nanoparticle films," Phys. Rev. Lett., vol. 111, no. 13, p. 136808, 2013.

[40] S. Fostner and S. A. Brown, "Neuromorphic behavior in percolating nanoparticle films," Phys. Rev. E, vol. 92, no. 5, p. $052134,2015$.

[41] A. Z. Stieg, A. V. Avizienis, H. O. Sillin, C. Martin-Olmos, M. L. Lam, M. Aono, and J. K. Gimzewski, "Self-organized atomic switch networks," Jpn. J. Appl. Phys., vol. 53, p. 01AA02, 2014.

[42] E. T. Rolls and G. Deco, The Noisy Brain: Stochastic dynamics as a principle of brain function. Oxford University Press, 2010.

[43] R. Reichel, J. G. Partridge, A. D. F. Dunbar, S. A. Brown, O. Caughley, and A. Ayesh, "Construction and Application of a UHV Compatible Cluster Deposition System,” J. Nanoparticle Res., vol. 8, no. 3-4, pp. 405-416, 2006.

[44] A. D. F. Dunbar, J. G. Partridge, M. Schulze, and S. A. Brown, "Morphological differences between Bi, Ag and Sb nano-particles and how they affect the percolation of current through nano-particle networks," Eur. Phys. J. D, vol. 39, no. 3, pp. 415-422, 2006.

[45] A. I. Ayesh, A. Lassesson, S. A. Brown, A. D. F. Dunbar, M. Kaufmann, J. G. Partridge, R. Reichel, and J. van Lith, "Experimental and simulational study of the operation conditions for a high transmission mass filter," Rev. Sci. Instrum., vol. 78, no. 5, p. 053906, 2007.

[46] D. Stauffer and A. Aharony, Introduction to percolation theory, 2nd ed. New York: CRC Press, 1992.

[47] D. R. Chialvo, "Emergent complex neural dynamics," Nat. Phys., vol. 6, no. 10, pp. 744-750, 2010.

[48] X. Yu, P. M. Duxbury, G. Jeffers, and M. A. Dubson, "Coalescence and percolation in thin metal films," Phys. Rev. B, vol. 44, no. 23, pp. $13163-13166,1991$.

[49] B. J. van Wees, H. van Houten, C. W. J. Beenakker, J. G. Williamson, L. P. Kouwenhoven, D. van der Marel, and C. T. Foxon, "Quantized conductance of point contacts in a two-dimensional electron gas," Phys. Rev. Lett., vol. 60, no. 9, pp. 848-850, 1988.

[50] D. Moldovan, V. Yamakov, D. Wolf, and S. R. Phillpot, "Scaling behavior of grain-rotation-induced grain growth." Phys. Rev. Lett., vol. 89, no. 20, p. 206101, 2002.

[51] M. Olsen, M. Hummelgård, and H. Olin, "Surface modifications by field induced diffusion," PLoS One, vol. 7, no. 1, p. e30106, 2012.

[52] C. Xiang, J. Y. Kim, and R. M. Penner, "Reconnectable sub-5 nm nanogaps in ultralong gold nanowires," Nano Lett., vol. 9, no. 5, pp. 2133-2138, 2009.

[53] S. Cho, J. Yu, S. K. Kang, and D.-Y. Shih, "Oxidation study of pure tin and its alloys via electrochemical reduction analysis," J. Electron. Mater., vol. 34, no. 5, pp. 635-642, 2005.

[54] E. Sutter, F. Ivars-Barcelo, and P. Sutter, "Size-dependent room temperature oxidation of tin particles," Part. Part. Syst. Charact., vol. 31, no. 8, pp. 879-885, 2014.

[55] A. F. Lee and R. M. Lambert, "Oxidation of Sn overlayers and the structure and stability of Sn oxide films on Pd(111)," Phys. Rev. $B$, vol. 58, no. 7, pp. 4156-4165, 1998.

[56] A. A. Faisal, L. P. J. Selen, and D. M. Wolpert, "Noise in the nervous system." Nat. Rev. Neurosci., vol. 9, pp. $292-303,2008$.

[57] G. M. Hoerzer, R. Legenstein, and W. Maass, "Emergence of complex computational structures from chaotic neural networks through reward-modulated hebbian learning," Cereb. Cortex, vol. 24, no. 3, pp. 677-690, 2014.

[58] J. G. Simmons, "Generalized Formula for the Electric Tunnel Effect between Similar Electrodes Separated by a Thin Insulating Film," J. Appl. Phys., vol. 34, no. 6, pp. 1793-1803, 1963.

[59] N. Agraït, A. L. Yeyati, and J. M. van Ruitenbeek, "Quantum properties of atomic-sized conductors," Phys. Rep., vol. 377, no. 2-3, pp. 81-279, 2003.

[60] T. Hasegawa, T. Ohno, K. Terabe, T. Tsuruoka, T. Nakayama, J. K. Gimzewski, and M. Aono, "Learning abilities achieved by a single solid-state atomic switch," Adv. Mater., vol. 22, no. 16, pp. 1831-1834, 2010. 
[61] T. Ohno, T. Hasegawa, A. Nayak, T. Tsuruoka, J. K. Gimzewski, and M. Aono, "Sensory and short-term memory formations observed in a Ag2S gap-type atomic switch," Appl. Phys. Lett., vol. 99, no. 20, p. 203108, 2011.

[62] T. Chang, S. H. Jo, and W. Lu, "Short-term memory to long-term memory transition in a nanoscale memristor," ACS Nano, vol. 5, no. 9, pp. 7669-7676, 2011.

[63] N. Onofrio, D. Guzman, and A. Strachan, "Atomic origin of ultrafast resistance switching in nanoscale electrometallization cells," Nat. Mater., vol. 14, no. 4, pp. 440-446, 2015.

[64] M. Lukoševičius and H. Jaeger, "Reservoir computing approaches to recurrent neural network training," Comput. Sci. Rev., vol. 3, no. 3, pp. 127-149, 2009.

[65] L. Büsing, B. Schrauwen, and R. Legenstein, "Connectivity, dynamics, and memory in reservoir computing with binary and analog neurons." Neural Comput., vol. 22, no. 5, pp. 1272-1311, 2010. 